\title{
In vitro Protocol for Direct Organogenesis in Momordica cymbalaria. Fenzl
}

\author{
T. Devi, V. Rajasree*, V. Premalakshmi, K. Hemaprabha and S. Praneetha \\ Department of Vegetable Crops, HC \& RI, Tamil Nadu Agricultural University, \\ Coimbatore - 641003, Tamil Nadu, India \\ *Corresponding author
}

\section{A B S T R A C T}

\section{Keywords}

M. cymbalaria, Micropropagation, Direct organonesis, Shoot regeneration, Rooting, Hardening

Article Info

Accepted:

20 March 2017

Available Online:

10 April 2017
Momordica cymbalaria Fenzl. is a perennial herbaceous climber belongs to cucurbitaceae family and the genus of bitter gourd (Momordica charantia). Fruits are used as vegetables, it possess hypoglycaemic, hypolipidemic, cardio protective, hepatoprotective, nephroprotective and antioxidant properties. It is rich in crude fiber $(6.42 \mathrm{~g} / 100 \mathrm{~g})$, calcium $(72 \mathrm{~g} / 100 \mathrm{~g})$, potassium, sodium and vitamin C (290 g/100g). The major constraints in propagation of Momordica cymbalaria are non-availability of standardized propagation methods and low yield. In vitro propagation technique could be a valuable alternative to conventional methods for mass multiplication. To achieve this goal, investigation on micropropagation of Momordica cymbalaria was carried out at the Tissue Culture Laboratory of Horticultural College and Research Institute, TNAU Coimbatore. For direct organogenesis, MS medium supplemented with $2.0 \mathrm{mgl}^{-1}$ of BAP and $3.0 \mathrm{mgl}^{-1}$ of kinetin was found to be the most suitable concentration for maximum shoot regeneration percentage and shoot length for both axillary bud and shoot tip. Efficient rooting was observed in half MS medium fortified with $1.0 \mathrm{mgl}^{-1} \mathrm{IBA}+0.1 \mathrm{mgl}^{-1}$ of NAA. Rooted plantlets were hardened with various media among which the sterilized pot mixture, coco peat was found to be the most ideal hardening medium. The present study revealed that micro propagation through direct organogenesis using axillary bud could serve as a viable protocol, when MS medium is augmented with $2.0 \mathrm{mgl}^{-1} \mathrm{BAP}$ is used for shoot regeneration. These shoots multiplied to 7.48 numbers in MS medium supplemented with $3.0 \mathrm{mgl}^{-1} \mathrm{BAP}$, followed by maximum rooting percentage in half MS medium fortified with $1.0 \mathrm{mgl}^{-1} \mathrm{IBA}+0.1 \mathrm{mgl}^{-1}$ of NAA and finally acclimatized in pot mixture, coco peat which afford mass multiplication of Momordica cymbalarias.

\section{Introduction}

Momordica cymbalaria Fenzl. is a perennial herbaceous climber, which belongs to cucurbitaceae family and the genus of bitter gourd (Momordica charantia). It is widely distributed in the tropical regions of Africa and India. It is found in the south Indian states of Andhra Pradesh, Karnataka, Madhya Pradesh, Maharashtra and Tamil Nadu. Fruits are used as vegetables it possess hypoglycaemic, hypolipidemic, cardio protective, hepatoprotective, nephroprotective and antioxidant properties (Jeyadevi et al., 2012). The roots of this plant have been used by the natives of north Karnataka and Andhra Pradesh to treat some gynecological ailments and also to induce abortions. The tuber is used as an abortifacient (Parvathi and Kumar 2002). 
The minimum requirement of vegetable consumption is $280 \mathrm{~g} /$ day/adult. However in many developing countries vegetable consumption is far below the normal requirement, which may be attributed to the low availability or unaffordable cost. The feasible alternative is to explore additional source of food and to minimize the load on production of conventional food plants. The fruits consumed as vegetable are rich in crude fiber $(6.42 \mathrm{~g} / 100 \mathrm{~g})$, calcium $(72 \mathrm{~g} / 100 \mathrm{~g})$, potassium, sodium and vitamin $\mathrm{C}$ $(290 \mathrm{~g} / 100 \mathrm{~g})$ as compared to bitter gourd and its fruits have medicinal value. The major constraints in propagation of Momordica cymbalaria are non-availability of standardized propagation methods and low yield. A limited number of perennial tubers survive in soil and produce single plant in the next season. The population is reduced by consumption of tubers by animals and water logging conditions, respectively. The fruits had few seeds that remain dormant and low germination rate further curtails the propagation through seeds. It can be overcome by biotechnological approach through micropropagation.

To achieve this goal, the first important step is to establish a reliable tissue culture system for plant regeneration. The controlled conditions provide the culture an environment conducive for growth and multiplication. These conditions include proper supply of nutrients, $\mathrm{pH}$ medium, adequate temperature, proper light environment. Plant tissue culture technology is being widely used for large scale plant multiplication. Endangered, threatened and rare species have successfully been grown and conserved by micro propagation because of high coefficient of multiplication and small demands on number of initial plants and space. There has been progress in tissue culture studies in many cucurbitaceae members such as Venkateshwaralu (2009) in Coccinia indica, Karim and Ahmed (2010) in Momordica dioica, Ugandhar et al., 2011 in Cucumis sativus, Jeyakumar et al.,2013 in Cucumis angurina, Debnath et al., (2013) and Rathod (2015) in Momordica dioica.

To date there is not much reports on tissue culture system of Momordica cymbalaria. Hence the present study was under taken to standardize the suitable explants for micro propagation and suitable culture media for in vitro shoot regeneration and rooting of shoots.

\section{Materials and Methods}

Tuber materials were collected from the farmer's field in Ramanathapuram, planted in college orchard, Tamil Nadu agricultural university, Coimbatore for explant collection. The explants used were nodal segments and shoot tip. The explants were washed with teepol to remove the soil debris and pretreated with $0.2 \%$ bavistin and $0.1 \%$ streptomycin for $30 \mathrm{~min}$ on rotatory shaker and thoroughly washed with distilled water. The explants were then surface sterilized by using $70 \%$ ethanol for $30 \mathrm{sec}$. followed by $0.2 \% \mathrm{HgCl}_{2}$ for $5 \mathrm{~min}$. The explants were then washed with sterile distilled water to remove trace of mercuric chloride.

The axillary bud and shoot tip were placed on MS medium basal medium (Murashige and Skoog, 1962) with 3\% sucrose was used for breaking the dormancy of shoot buds as well as for proliferation. The $\mathrm{pH}$ of the medium was adjusted to 5.7 using $0.1 \mathrm{~N} \mathrm{KOH}$ or $0.1 \mathrm{~N}$ $\mathrm{HCl}$ as and when required and $0.7 \%(\mathrm{w} / \mathrm{v})$ agar (Merck) before autoclaving. Approximately $20 \mathrm{ml}$ of molten medium was dispensed into each culture tube $(25 \times 150$ $\mathrm{mm}$ ) and plugged with non-absorbent cotton. The tubes steam sterilized at $15 \mathrm{lb}$ inch 20 min. All the cultures were grown at $24 \pm 2{ }^{\circ} \mathrm{C}$ temperature and $55 \pm 5 \%$ relative humidity under $16 \mathrm{~h}$ photoperiod supplied by Philips fluorescent tubes. 


\section{In vitro shoot regeneration and multiplication}

For in vitro shoot multiplication, the explants were cultured on MS medium alone as control and MS medium with different concentrations of cytokinins (BAP, Kn). The effects of different plant growth regulators were tested on multiple shoot induction, shoot elongation, and were recorded periodically.

\section{In vitro root induction}

For rooting, in vitro grown shoots with more than four leaves were excised and implanted on half MS basal media and with different concentration of IBA and NAA. The cultures were examined every week and were recorded on the basis of visual observations.

\section{Hardening of plants}

For hardening, at first the tissue culture derived healthy rooted plantlets were removed from the agar medium, washed thoroughly under running tap water and transferred to earthen pots containing sterilized media composition. To preserve moisture, the potted plantlets were covered with transparent polythene bag and placed in the green house. After one month, plantlets were transferred to the main field.

\section{Statistical analysis}

The experiment design was Completely Randomized block Design and Factorial (FCRD) as per the standard procedures of Gomez and Gomez (1984). Observations recorded as percentage were subjected to angular transformation (Snedecor and Cochran, 1980). Analysis was carried out with AGRES software package and MS Excel spreadsheet. The means of different experiments were compared using DMRT (Duncan's Multiple Range Test) at the 5\% level.

\section{Results and Discussion}

\section{Effect of different concentrations of (BAP)} cytokinin on shoot regeneration

Table 1 represents the shoot regeneration of nodal segment, shoot tip explant to various concentrations of BAP and $\mathrm{Kn}$. It was observed that maximum response was observed in nodal segment explants compared to shoot tip in both the cytokinins Minimum days (4.60) was taken for bud sprout in MS medium supplemented with $3.0 \mathrm{mg} / \mathrm{l}$ of BAP followed by $2.5 \mathrm{mg} / \mathrm{l}$ of BAP when nodal segment used as explant and maximum days (19.4 days) taken for bud sprout was recorded in control. Maximum shoot length was observed in MS augmented with $2.5 \mathrm{mg} / \mathrm{l}$ of BAP $(5.62 \mathrm{~cm})$ followed by MS supplemented with $2.0 \mathrm{mg} / 1$ of BAP $(5.46 \mathrm{~cm})$. The maximum shoot regeneration percentage (97.7\%) was recorded in MS medium supplemented with $2.0 \mathrm{mg} / \mathrm{l}$ of BAP followed by MS medium fortified with $2.5 \mathrm{mg} / \mathrm{l}$ of BAP $(95.5 \%)$ and minimum shoot regeneration percentage was observed in MS basal medium $(12.5 \%)$.

\section{Effect of different concentration of $\mathrm{Kn}$ on shoot regeneration}

It was observed that minimum days (4.46) taken for bud sprouting was observed in MS medium enriched with $3.0 \mathrm{mg} / \mathrm{l}$ of $\mathrm{Kn}$ and the maximum days (8.23) taken for bud sprout was recorded in control. Maximum shoot length (6.76) and maximum shoot induction percentage $(75.3 \%)$ was observed in MS medium supplemented with $3.0 \mathrm{mg} / \mathrm{l}$ of $\mathrm{Kn}$. The MS basal medium showed less response (Table 3).

In the present study, MS medium supplemented with $3.0 \mathrm{mgl}^{-1}$ of BAP recorded the minimum number of days taken for bud emergence for both shoot tip and axillary bud 
explant followed by the MS medium supplemented with $2.5 \mathrm{mgl}^{-1}$ of BAP. It may due to the effect of cytokinin to overcome apical dominance, release lateral buds from dormancy, and promote shoot formation. The results are in conformity with findings of Mahzabin et al., 2008 in Cucurbita maxima, Ugandhar et al., (2011) in Cucumis sativus, Rani et al., 2012 in Murraya koenigii, Munsur et al., (2007) in Momordica charantia. The shoot length was higher in MS medium supplemented with $2.5 \mathrm{mgl}^{-1}$ of BAP followed by MS medium supplemented with $2.0 \mathrm{mgl}^{-1}$ of BAP. But interestingly shoot induction percentage was higher in MS+2.0 mgl ${ }^{-1}$ of BAP. This may due to increasing concentration of BAP cause antagonistic effect. It could be inferred from above results that $2.0 \mathrm{mgl}^{-1}$ of BAP is the optimum concentration for the better performance of this particular hormone. Any high or low from this normal concentration of the growth regulator exacerbated the response. The fact that higher doses failed to manifest their effect could be attributed to an obnoxious effect at higher concentration, whereas, the ineffectiveness of the lower dose indicated inadequate dose of hormone as a consequence indicating poor performance. These findings can be affirmatively compared with the observations of Ugandhar et al., (2011) in cucumber and Venkeshwaralu (2012) in Cucumis melo. Many literatures suggest that BAP is most active at the concentration of 1.0 to $2.0 \mathrm{mgl}^{-1}$ in many plant species (Senthilkumar et al., 2007; Karupusamy et al., 2006; Arulanandam et al., 2011).

Table.1 Effect of different concentrations of BAP on shooting in Momordica cymbalaria

\begin{tabular}{|c|c|c|c|c|c|c|c|}
\hline \multirow[b]{2}{*}{$\begin{array}{l}\text { Treatme } \\
\text { nt code }\end{array}$} & \multirow{2}{*}{$\begin{array}{l}\text { Treatment } \\
\text { details }\end{array}$} & \multicolumn{2}{|c|}{$\begin{array}{l}\text { Days taken for bud } \\
\text { sprout }\end{array}$} & \multicolumn{2}{|c|}{ Length of shoot $(\mathrm{cm})$} & \multicolumn{2}{|c|}{$\begin{array}{l}\text { Percentage of shoot } \\
\text { induction }(\%)\end{array}$} \\
\hline & & $\begin{array}{l}\text { Axillary } \\
\text { bud }\left(E_{1}\right)\end{array}$ & $\begin{array}{l}\text { Shoot tip } \\
\left(\mathrm{E}_{2}\right)\end{array}$ & $\begin{array}{l}\text { Axillary } \\
\text { bud }\left(E_{1}\right)\end{array}$ & $\begin{array}{l}\text { Shoot } \\
\text { tip }\left(\mathrm{E}_{2}\right)\end{array}$ & $\begin{array}{l}\text { Axillary } \\
\text { bud }\left(E_{1}\right)\end{array}$ & $\begin{array}{l}\text { Shoot tip } \\
\left(\mathrm{E}_{2}\right)\end{array}$ \\
\hline $\mathrm{T}_{0}$ & MS media & $19.4^{\mathrm{f}}$ & $15.4^{\mathrm{g}}$ & $2.83^{\mathrm{f}}$ & $2.40^{\mathrm{g}}$ & $\begin{array}{l}16.5^{\mathrm{g}} \\
(23.9)\end{array}$ & $\begin{array}{l}12.5^{\mathrm{g}} \\
(20.7)\end{array}$ \\
\hline $\mathrm{T} 1$ & $\begin{array}{c}\mathrm{MS}+\mathrm{BAP} 1.0 \\
\mathrm{mgl}^{-1}\end{array}$ & $8.23^{\mathrm{e}}$ & $7.06^{\mathrm{d}}$ & $5.02^{\mathrm{b}}$ & $4.18^{\mathrm{e}}$ & $\begin{array}{l}89.9^{c} \\
(71.7)\end{array}$ & $\begin{array}{l}34.4^{t} \\
(35.9)\end{array}$ \\
\hline $\mathrm{T}_{2}$ & $\begin{array}{c}\mathrm{MS}+\mathrm{BAP} 1.5 \\
\mathrm{mgl}^{-1}\end{array}$ & $7.86^{\mathrm{de}}$ & $6.70^{\mathrm{d}}$ & $5.16^{\mathrm{b}}$ & $4.29^{\mathrm{e}}$ & $\begin{array}{l}92.2^{\mathrm{bc}} \\
(74.2)\end{array}$ & $\begin{array}{l}38.8^{\mathrm{e}} \\
(42.4)\end{array}$ \\
\hline $\mathrm{T}_{3}$ & $\begin{array}{c}\mathrm{MS}+\mathrm{BAP}^{-1} 2.0 \\
\mathrm{mgl}^{-1}\end{array}$ & $6.00^{\mathrm{bc}}$ & $6.13^{\mathrm{c}}$ & $5.46^{\mathrm{a}}$ & $4.62^{\mathrm{d}}$ & $\begin{array}{l}97.7^{\mathrm{a}} \\
(82.7)\end{array}$ & $\begin{array}{l}52.2^{\mathrm{d}} \\
(49.4)\end{array}$ \\
\hline $\mathrm{T}_{4}$ & $\begin{array}{c}\mathrm{MS}+\mathrm{BAP} \\
\mathrm{mgl}^{-1}\end{array}$ & $5.56^{\mathrm{b}}$ & $5.43^{b}$ & $5.62^{\mathrm{a}}$ & $4.73^{\mathrm{cd}}$ & $\begin{array}{l}95.5^{\mathrm{ab}} \\
(77.9)\end{array}$ & $\begin{array}{l}49.9^{d} \\
(47.5)\end{array}$ \\
\hline $\mathrm{T}_{5}$ & $\begin{array}{c}\mathrm{MS}+\mathrm{BAP} 3.0 \\
\mathrm{mgl}^{-1}\end{array}$ & $4.60^{\mathrm{a}}$ & $4.73^{\mathrm{a}}$ & $4.66^{\mathrm{bc}}$ & $4.97^{\mathrm{bc}}$ & $\begin{array}{l}45.5^{\mathrm{e}} \\
(42.4)\end{array}$ & $\begin{array}{c}27.7^{\mathrm{f}} \\
(33.8)\end{array}$ \\
\hline
\end{tabular}

(Note values in parenthesis are the angular transformed values of the original percentile data 
Int.J.Curr.Microbiol.App.Sci (2017) 6(4): 2392-2402

Table.2 Effect of different concentrations of kinetin on shooting in Momordica cymbalaria

\begin{tabular}{|c|c|c|c|c|c|c|c|}
\hline \multirow{2}{*}{$\begin{array}{l}\text { Treatment } \\
\text { code }\end{array}$} & \multirow[t]{2}{*}{$\begin{array}{c}\text { Treatment } \\
\text { details }\end{array}$} & \multicolumn{2}{|c|}{$\begin{array}{l}\text { Days taken for bud } \\
\text { sprout }\end{array}$} & \multicolumn{2}{|c|}{ Length of shoot $(\mathrm{cm})$} & \multicolumn{2}{|c|}{$\begin{array}{c}\text { Shoot induction percent } \\
(\%)\end{array}$} \\
\hline & & $\begin{array}{l}\text { Axillary } \\
\text { bud }\left(E_{1}\right)\end{array}$ & $\begin{array}{l}\text { Axillary bud } \\
\left(\mathrm{E}_{1}\right)\end{array}$ & $\begin{array}{l}\text { Axillary } \\
\text { bud }\left(E_{1}\right)\end{array}$ & $\begin{array}{l}\text { Axillary bud } \\
\left(\mathrm{E}_{1}\right)\end{array}$ & $\begin{array}{l}\text { Axillary } \\
\text { bud }\left(E_{1}\right)\end{array}$ & $\begin{array}{l}\text { Axillary bud } \\
\left(\mathrm{E}_{1}\right)\end{array}$ \\
\hline $\mathrm{T}_{0}$ & MS medium & $19.4^{\mathrm{i}}$ & $15.4^{\mathrm{h}}$ & 2.80 & 2.43 & $23.9^{\mathrm{h}}$ & $20.7^{\mathrm{h}}$ \\
\hline $\mathrm{T} 1$ & $\begin{array}{c}\mathrm{MS}+\mathrm{KN} \\
\mathrm{mgl}^{-1}\end{array}$ & $8.00^{\mathrm{g}}$ & $6.10^{\mathrm{e}}$ & 3.94 & 3.90 & $60.4^{c}$ & $31.7^{\mathrm{g}}$ \\
\hline $\mathrm{T}_{2}$ & $\begin{array}{c}\mathrm{MS}+\mathrm{KN} \\
1.5 \mathrm{mgl}^{-1}\end{array}$ & $7.20^{\mathrm{f}}$ & $5.83^{\mathrm{de}}$ & 5.31 & 4.71 & $64.1^{\mathrm{bc}}$ & $33.8^{\mathrm{fg}}$ \\
\hline $\mathrm{T}_{3}$ & $\begin{array}{c}\mathrm{MS}+\mathrm{KN} \\
2.0 \mathrm{mgl}^{-1}\end{array}$ & $5.80^{\mathrm{de}}$ & $5.40^{\mathrm{cd}}$ & 5.86 & 5.17 & $65.7^{\mathrm{b}}$ & $37.9^{\mathrm{ef}}$ \\
\hline $\mathrm{T}_{4}$ & $\begin{array}{c}\mathrm{MS}+\mathrm{KN} \\
2.5 \mathrm{mgl}^{-1}\end{array}$ & $5.33^{\mathrm{cd}}$ & $5.00^{\mathrm{bc}}$ & 6.30 & 5.53 & $72.7^{\mathrm{a}}$ & $41.1^{\mathrm{de}}$ \\
\hline $\mathrm{T}_{5}$ & $\begin{array}{c}\mathrm{MS}+\mathrm{KN} \\
3.0 \mathrm{mgl}^{-1}\end{array}$ & $4.46^{\mathrm{a}}$ & $4.60^{\mathrm{ab}}$ & 6.76 & 5.92 & $75.3^{\mathrm{a}}$ & $43.0^{\mathrm{d}}$ \\
\hline
\end{tabular}

Table.3 Effect of different concentrations of BAP on shooting in Momordica cymbalaria

\begin{tabular}{|c|c|c|c|c|c|c|c|c|c|}
\hline \multirow[t]{2}{*}{$\begin{array}{l}\text { Treatme } \\
\text { nt code }\end{array}$} & \multirow[t]{2}{*}{$\begin{array}{l}\text { Treatme } \\
\text { nt details }\end{array}$} & \multicolumn{2}{|c|}{$\begin{array}{l}\text { Days taken for } \\
\text { multiple shoot } \\
\text { induction }\end{array}$} & \multicolumn{2}{|c|}{$\begin{array}{l}\text { Length of shoot } \\
(\mathrm{cm})\end{array}$} & \multicolumn{2}{|c|}{$\begin{array}{c}\text { No. of shoots per } \\
\text { explant }\end{array}$} & \multicolumn{2}{|c|}{$\begin{array}{l}\text { Percentage of } \\
\text { multiple shoot } \\
\text { induction }\end{array}$} \\
\hline & & $\begin{array}{l}\text { Axillary } \\
\text { bud }\left(E_{1}\right)\end{array}$ & $\begin{array}{l}\text { Axillary } \\
\text { bud }\left(E_{1}\right)\end{array}$ & $\begin{array}{l}\text { Axillary } \\
\text { bud }\left(E_{1}\right)\end{array}$ & $\begin{array}{l}\text { Axillary } \\
\text { bud }\left(E_{1}\right)\end{array}$ & $\begin{array}{l}\text { Axillary } \\
\text { bud }\left(E_{1}\right)\end{array}$ & $\begin{array}{l}\text { Axillary } \\
\text { bud }\left(E_{1}\right)\end{array}$ & $\begin{array}{l}\text { Axillary } \\
\text { bud }\left(E_{1}\right)\end{array}$ & $\begin{array}{l}\text { Axillary } \\
\text { bud }\left(E_{1}\right)\end{array}$ \\
\hline $\mathrm{T}_{0}$ & $\begin{array}{c}\text { MS } \\
\text { medium }\end{array}$ & $61.4^{\mathrm{g}}$ & $66.5^{\mathrm{h}}$ & 1.51 & 1.44 & $1.26^{\mathrm{i}}$ & $0.80^{\mathrm{j}}$ & $\begin{array}{r}30.5^{\mathrm{i}} \\
(33.5) \\
\end{array}$ & $\begin{array}{l}27.1^{\mathrm{i}} \\
(31.4) \\
\end{array}$ \\
\hline $\mathrm{T} 1$ & $\begin{array}{c}\mathrm{MS}+ \\
\text { BAP 2.0 } \\
\mathrm{mgl}^{-1}\end{array}$ & $52.0^{\mathrm{f}}$ & $54.0^{\mathrm{e}}$ & 4.00 & 3.53 & $5.51^{\mathrm{c}}$ & $2.30^{\mathrm{g}}$ & $\begin{array}{l}77.7^{\mathrm{c}} \\
(61.8) \\
\end{array}$ & $\begin{array}{r}53.3^{\mathrm{g}} \\
(46.9) \\
\end{array}$ \\
\hline $\mathrm{T}_{2}$ & $\begin{array}{c}\mathrm{MS}+ \\
\mathrm{BAP} 2.5 \\
\mathrm{mgl}^{-1}\end{array}$ & $47.4^{\mathrm{d}}$ & $48.2^{\mathrm{d}}$ & 4.76 & 4.50 & $6.49^{b}$ & $2.73^{\mathrm{f}}$ & $\begin{array}{l}86.6^{\mathrm{b}} \\
(68.6)\end{array}$ & $\begin{array}{l}58.4^{\mathrm{fg}} \\
(49.8)\end{array}$ \\
\hline $\mathrm{T}_{3}$ & $\begin{array}{c}\mathrm{MS}+ \\
\text { BAP } 3.0 \\
\mathrm{mgl}^{-1}\end{array}$ & $34.5^{\mathrm{c}}$ & $36.0^{\mathrm{c}}$ & 6.13 & 6.05 & $7.48^{\mathrm{a}}$ & $4.20^{\mathrm{d}}$ & $\begin{array}{l}93.3^{\mathrm{a}} \\
(75.3) \\
\end{array}$ & $\begin{array}{l}61.1^{\mathrm{ef}} \\
(51.4)\end{array}$ \\
\hline $\mathrm{T}_{4}$ & $\begin{array}{c}\mathrm{MS}+ \\
\mathrm{BAP}^{3.5} \\
\mathrm{mgl}^{-1}\end{array}$ & $31.8^{\mathrm{b}}$ & $32.5^{\mathrm{b}}$ & 2.53 & 2.00 & $3.53^{\mathrm{e}}$ & $2.00^{\mathrm{g}}$ & $\begin{array}{l}72.2^{\mathrm{d}} \\
(58.2)\end{array}$ & $\begin{array}{l}45.5^{\mathrm{h}} \\
(42.4)\end{array}$ \\
\hline $\mathrm{T}_{5}$ & $\begin{array}{c}\mathrm{MS}+ \\
\mathrm{BAP} 4.0 \\
\mathrm{mgl}^{-1}\end{array}$ & $30.2^{\mathrm{a}}$ & $30.9^{\mathrm{ab}}$ & 1.83 & 1.63 & $2.26^{\mathrm{g}}$ & $1.86^{\mathrm{h}}$ & $\begin{array}{l}64.4^{\mathrm{e}} \\
(53.4) \\
\end{array}$ & $\begin{array}{l}42.5^{\mathrm{h}} \\
(40.7) \\
\end{array}$ \\
\hline
\end{tabular}

(Note: Values in parenthesis are the angular transformed values of the original percentile data) 
Table.4 Effect of different concentrations of IBA and NAA on rooting

\begin{tabular}{|c|c|c|c|c|c|}
\hline $\begin{array}{l}\text { Treatment } \\
\text { code }\end{array}$ & Treatment details & $\begin{array}{l}\text { No of Days } \\
\text { taken for root } \\
\text { induction }\end{array}$ & $\begin{array}{l}\text { No of } \\
\text { roots per } \\
\text { plant }\end{array}$ & $\begin{array}{l}\text { Root } \\
\text { length }\end{array}$ & $\begin{array}{c}\text { Root } \\
\text { induction } \\
\text { percentage }\end{array}$ \\
\hline $\mathrm{T}_{0}$ & Half MS media & $24.9^{f}$ & $2.33^{\mathrm{h}}$ & $0.50^{\mathrm{g}}$ & $\begin{array}{c}34.0^{1} \\
(34.7)\end{array}$ \\
\hline $\mathrm{T} 1$ & $\begin{array}{c}\text { Half MS+ IBA } 0.5 \mathrm{mg} \\
\mathrm{l}^{-1}\end{array}$ & $10.5^{\mathrm{e}}$ & $6.37^{\mathrm{g}}$ & $0.67^{g}$ & $\begin{array}{r}63.4^{\mathrm{h}} \\
(52.8)\end{array}$ \\
\hline$T_{2}$ & $\begin{array}{l}\text { Half MS+ IBA } 0.5+ \\
\text { NAA } 0.1 \mathrm{mg} \mathrm{l}^{-1}\end{array}$ & $8.27^{\mathrm{d}}$ & $7.30^{\mathrm{fg}}$ & $1.33^{\mathrm{f}}$ & $\begin{array}{r}66.8^{\mathrm{g}} \\
(54.8)\end{array}$ \\
\hline $\mathrm{T}_{3}$ & $\begin{array}{l}\text { Half MS+ IBA } 0.5+ \\
\text { NAA } 0.2 \mathrm{mg} \mathrm{l}^{-1}\end{array}$ & $8.13 \mathrm{c}^{\mathrm{d}}$ & $7.80^{\mathrm{fg}}$ & $1.80^{\mathrm{e}}$ & $\begin{array}{r}73.4^{\mathrm{f}} \\
(58.9)\end{array}$ \\
\hline $\mathrm{T}_{4}$ & $\begin{array}{l}\text { Half MS+ IBA } 0.5+ \\
\quad \text { NAA } 0.3 \mathrm{mg} \mathrm{l}^{-1}\end{array}$ & $7.90 c^{d}$ & $8.20^{\mathrm{f}}$ & $2.20^{\mathrm{d}}$ & $\begin{array}{r}77.7^{\mathrm{e}} \\
(61.8)\end{array}$ \\
\hline $\mathrm{T}_{5}$ & $\begin{array}{l}\text { Half MS+ IBA } 1.0+ \\
\text { NAA } 0.1 \mathrm{mg} \mathrm{l}^{-1}\end{array}$ & $7.57^{\mathrm{bc}}$ & $33.6^{\mathrm{a}}$ & $3.20^{b}$ & $\begin{array}{r}83.5^{\mathrm{c}} \\
(66.0) \\
\end{array}$ \\
\hline $\mathrm{T}_{6}$ & $\begin{array}{l}\text { Half MS+ IBA } 1.0+ \\
\quad \text { NAA } 0.2 \mathrm{mg} \mathrm{l}^{-1}\end{array}$ & $7.27^{b}$ & $33.3^{\mathrm{a}}$ & $3.57^{\mathrm{a}}$ & $\begin{array}{l}93.5^{\mathrm{a}} \\
(75.2)\end{array}$ \\
\hline $\mathrm{T}_{7}$ & $\begin{array}{l}\text { Half MS+ IBA } 1.0+ \\
\quad \text { NAA } 0.3 \mathrm{mg} \mathrm{l}^{-1}\end{array}$ & $7.17^{b}$ & $29.6^{\mathrm{b}}$ & $3.67^{\mathrm{a}}$ & $\begin{array}{r}85.9^{\mathrm{b}} \\
(67.9)\end{array}$ \\
\hline $\mathrm{T}_{8}$ & $\begin{array}{l}\text { Half MS+ IBA } 1.5+ \\
\text { NAA } 0.1 \mathrm{mg} \mathrm{l}^{-1}\end{array}$ & $5.30^{\mathrm{a}}$ & $25.0^{\mathrm{c}}$ & $1.90^{\mathrm{e}}$ & $\begin{array}{r}80.5^{\mathrm{d}} \\
(63.8)\end{array}$ \\
\hline $\mathrm{T}_{9}$ & $\begin{array}{l}\text { Half MS+ IBA } 1.5+ \\
\text { NAA } 0.2 \mathrm{mg} \mathrm{l}^{-1}\end{array}$ & $5.40^{\mathrm{a}}$ & $22.0^{\mathrm{d}}$ & $2.23^{\mathrm{d}}$ & $\begin{array}{l}75.9^{\mathrm{e}} \\
(60.6)\end{array}$ \\
\hline $\mathrm{T}_{10}$ & $\begin{array}{l}\text { Half MS+ IBA } 1.5+ \\
\text { NAA } 0.3 \mathrm{mg} \mathrm{l}^{-1}\end{array}$ & $5.20^{\mathrm{a}}$ & $19.0^{\mathrm{e}}$ & $2.14^{\mathrm{d}}$ & $\begin{array}{r}72.5^{\mathrm{f}} \\
(58.3) \\
\end{array}$ \\
\hline
\end{tabular}

(Note: values in parenthesis are the angular transformed values of the original percentile data) 
Table.5 Effect of hardening media on ex vitro plantlet of Momordica cymbalaria

\begin{tabular}{|c|c|c|c|c|}
\hline $\begin{array}{c}\text { Treatment } \\
\text { code }\end{array}$ & Treatment detail & $\begin{array}{c}\text { Ex vitro plantlet } \\
\text { survival }(\%)\end{array}$ & $\begin{array}{c}\text { Days for emergence of } \\
1^{\text {st }} \text { new leaf }\end{array}$ & $\begin{array}{c}\text { Number of new } \\
\text { leaves }\end{array}$ \\
\hline $\mathrm{H} 1$ & $\begin{array}{c}\text { Pot mix } \\
(1: 1: 1 \text { of soil: sand: } \\
\text { FYM })\end{array}$ & $\begin{array}{c}91.2^{\mathrm{b}} \\
(72.7)\end{array}$ & $18.0^{\mathrm{b}}$ & $5.62^{\mathrm{b}}$ \\
\hline $\mathrm{H}_{2}$ & Sand & $\begin{array}{c}44.7^{\mathrm{d}} \\
(41.9)\end{array}$ & $21.6^{\mathrm{c}}$ & $2.61^{\mathrm{c}}$ \\
\hline $\mathrm{H}_{3}$ & Cocopeat & $\begin{array}{c}53.7^{\mathrm{c}} \\
(47.1)\end{array}$ & $25.0^{\mathrm{d}}$ & $1.30^{\mathrm{d}}$ \\
\hline $\mathrm{H}_{4}$ & $\begin{array}{c}\text { Cocopeat }+ \text { pot } \\
\text { mix }(1+1)\end{array}$ & $\begin{array}{c} \\
\left(77.0^{\mathrm{a}}\right.\end{array}$ & $16.0^{\mathrm{a}}$ & \\
& & $(77.0)$ & $6.30^{\mathrm{a}}$ \\
\hline
\end{tabular}

(Note: Values in parenthesis are the angular transformed values of the original percentile data) ** - High Significant

Figure.1 Effect of different concentrations of BAP on shoot multiplication

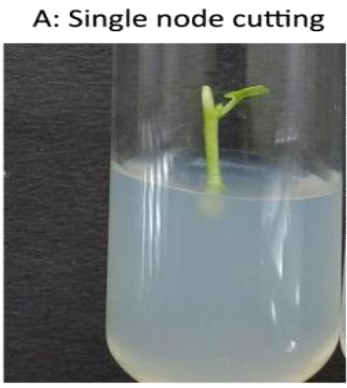

$\mathrm{C}: \mathrm{MS}+2.5 \mathrm{mgl}^{-1}$ of BAP

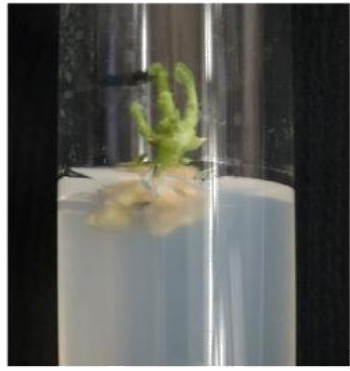

$\mathrm{E}: \mathrm{MS}+3.5 \mathrm{mgl}^{-1}$ of BAP

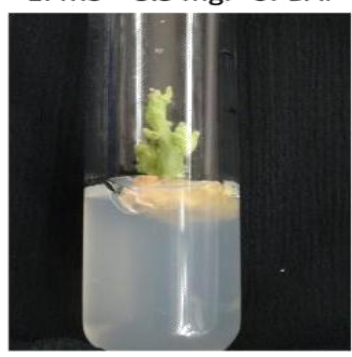

B: $\mathrm{MS}+2 \mathrm{mgl}^{-1}$ of BAP

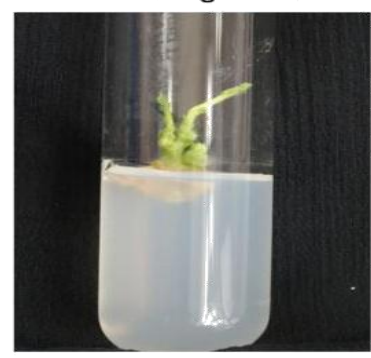

D: $\mathrm{MS}+3.0 \mathrm{mgl}^{-1}$ of BAP

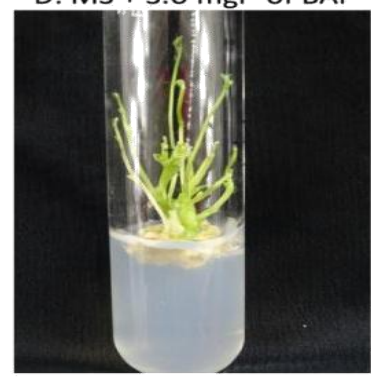

$\mathrm{F}: \mathrm{MS}+4.0 \mathrm{mgl}^{-1}$ of BAP

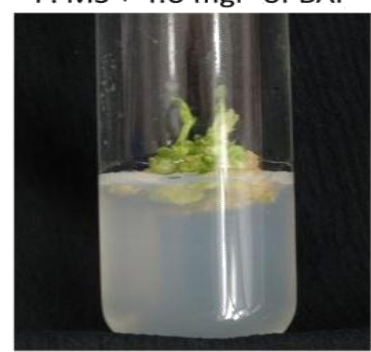


Figure.2 Effect of different concentrations of IBA and NAA on rooting

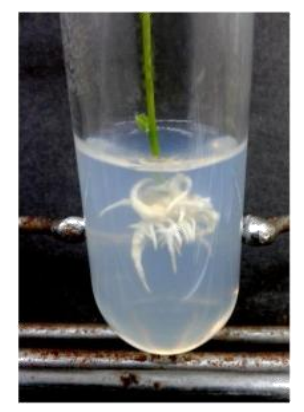

A: $1 / 2 \mathrm{MS}+0.5 \mathrm{mg}^{\mathrm{s}} \mathrm{IBA}$

$B: 1 / 2 \mathrm{MS}+1.0 \mathrm{mgl}^{-1} \mid \mathrm{BA}+0.2 \mathrm{mg}$

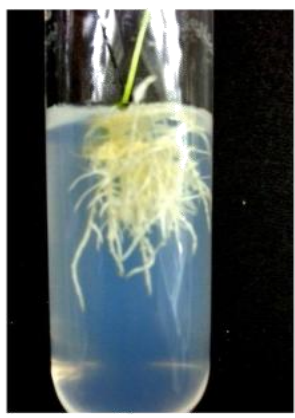

Figure.3 Hardening of in vitro grown M. cymbalaria

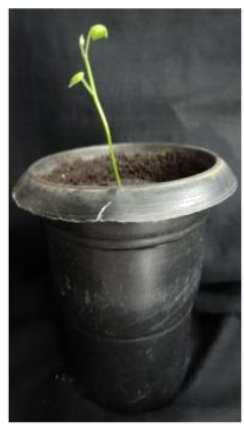

A: Hardened plant

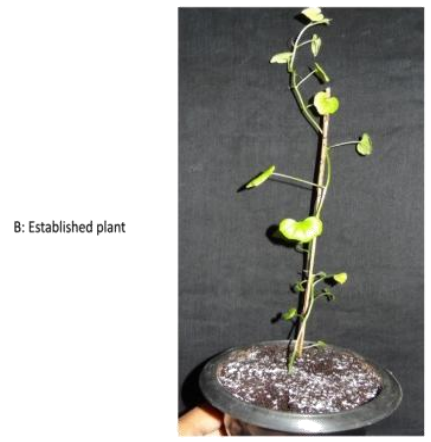




\section{Effect of different concentration of BAP on shoot multiplication}

Different concentration of BAP showed significant difference on shoot multiplication present in table 2 .

The minimum days (30.2) taken for multiple shoot induction was recorded in MS medium supplemented with $4.0 \mathrm{mg} / \mathrm{l}$ of BAP. Maximum multiple shoot induction percentage $(93.3 \%)$ and maximum number (7.48) of shoot per explant was recorded in MS medium fortified with $3.0 \mathrm{mg} / \mathrm{l}$ of BAP and minimum shoot induction percentage $(30.5 \%)$ and shoots (1.26) per explant was recorded in MS basal medium.

Among the two cytokinins, BAP proved to be better than kinetin in overall response of in vitro shoot regeneration of axillary bud and shoot tips of Momordica cymbalaria.

The above results are also in line with the reports Ugardhar et al., 2011 in Cucumis sativus. In the present study, the minimum number of days taken for multiple shoot induction was observed in MS supplemented with $4.0 \mathrm{mgl}^{-1}$ of BAP. Where Debnath et al., (2013) in Momordica dioica reported the same. But the main factors which directly influence the maximum shoot multiplication rate were shoot length, number of multiple shoot and multiple shoot induction percentage was maximum in $\mathrm{MS}+3.0 \mathrm{mgl}^{-1} \mathrm{BAP}$. Above this concentration number of multiple shoots, multiple shoot induction percentage gets reduced drastically. This may due to increasing the concentration of BAP in MS showed abnormality development of the buds. Such observations are in corroboration with those of Munsur et al., (2007) in Momordica charantia, Mahzabin et al., (2008) in Cucurbita maxima and Sridhar and Naidu (2011) in Solanum nigrum.

\section{Identification of viable explant}

Within the explants compared in the present study the axillary bud was found to be better and more convenient than shoot tip with respect to overall response for in vitro regeneration and shoot proliferation. This may due to the apical dominance of shoot tip which produce lesser shoot multiplication rate than axillary bud. The results of the present study are in corroboration with that of Verma et al., (2014).

\section{Effect of different concentration of NAA and IBA on rooting}

The in vitro rooting of regenerated shoot was presented in table 3 . The minimum of 5.20 days taken for root initiation was recorded in half MS medium supplemented with $1.5 \mathrm{mg} / \mathrm{l}$ of IBA+0.3 mg/l of NAA and maximum of 24.9 days taken for root initiation was observed in half MS basal medium. Maximum number (33.6) of roots per shoot was recorded in MS medium enriched with Half MS+ IBA 1.0+ NAA 0.1mg/l. Maximum root length of $3.67 \mathrm{~cm}$ was observed in MS medium enriched with Half MS+ IBA 1.0+ NAA $0.3 \mathrm{mg} / \mathrm{l}$ which was on par with Half MS+ IBA $1.0+$ NAA $0.2 \mathrm{mg} / \mathrm{l}(3.57 \mathrm{~cm})$. Maximum root induction percentage $(93.5 \%)$ was recorded in Half MS+ IBA $1.0+$ NAA $0.2 \mathrm{mg} / \mathrm{l}$ followed by Half MS+ IBA $1.0+$ NAA $0.3 \mathrm{mg} / \mathrm{l}$ showed $85.9 \%$ of root induction. Minimum root length $(0.50 \mathrm{~cm})$, least number of root (2.33) and minimum root induction percentage $(34.0 \%)$ was recorded in Half MS basal medium. The most commonly used auxin for root induction is IBA. In our present study, among the ten treatments minimum days taken for root induction were observed in $1 / 2 \mathrm{MS}$ medium $+1.5 \mathrm{mgl}^{-1} \mathrm{IBA}+$ $0.1 \mathrm{mgl}^{-1}$ NAA which were on par with $1 / 2 \mathrm{MS}$ medium $+1.5 \mathrm{mgl}^{-1} \mathrm{IBA}+0.2 \mathrm{mgl}^{-1} \mathrm{NAA}$ and $1 / 2$ MS medium $+1.5 \mathrm{mgl}^{-1} \mathrm{IBA}+0.3 \mathrm{mgl}^{-1}$ NAA. The number of root per shoot was 
higher in $1 / 2 \mathrm{MS}$ medium $+1.0 \mathrm{mgl}^{-1} \mathrm{IBA}+0.1$ $\mathrm{mgl}^{-1}$ NAA which were on par with $1 / 2 \mathrm{MS}$ medium $+1.0 \mathrm{mgl}^{-1} \mathrm{IBA}+0.2 \mathrm{mgl}^{-1} \mathrm{NAA}$.

The highest root length was observed in $1 / 2$ MS medium $+1.0 \mathrm{mgl}^{-1} \mathrm{IBA}+0.2 \mathrm{mgl}^{-1} \mathrm{NAA}$ which were on par with $1 / 2 \mathrm{MS}$ medium +1.0 $\mathrm{mgl}^{-1}$ IBA+ $0.3 \mathrm{mgl}^{-1}$ NAA. The percentage of root induction percentage was higher in $1 / 2 \mathrm{MS}$ medium $+1.0 \mathrm{mgl}^{-1} \mathrm{IBA}+0.2 \mathrm{mgl}^{-1} \mathrm{NAA}$ followed by $1 / 2 \mathrm{MS}$ medium $+1.0 \mathrm{mgl}^{-1} \mathrm{IBA}+$ $0.3 \mathrm{mgl}^{-1}$ NAA. Anisley and Lee (2010) found IBA is the best rooting hormone.

Contrarily, Reddy et al., (2012) in Asclepias curassavica, observed the highest number of root in $1 / 2$ MS medium supplemented with 1.0 $\mathrm{mgl}^{-1}+0.2 \mathrm{mgl}^{-1}$ kinetin. Anbazhagan et al., (2014), for rooting in banana $1 / 2$ MS medium supplemented with $1.0 \mathrm{mgl}^{-1}$ IBA. Pal et al., (2007) in summer squash observed $70 \%$ rooting in $1 / 2$ MS medium supplemented with $1.0 \mathrm{mgl}^{-1} \mathrm{IBA}$. The role of IBA in favoring the conjugation between endogenous IAA and amino acids that leads to the synthesis of the specific protein necessary for the formation of root initiation.

Effect of hardening media on ex vitro plantlet of Momordica cymbalaria

Effect of different media concentrations on hardening of in vitro grown Momordica cymbalaria was furnished in table 4 . Among the media concentration used, cocopeat + pot mix $(1+1)$ showed minimum days for emergence of $1^{\text {st }}$ new leaf and the maximum number of new leaves and survival percentage was also recorded in cocopeat + pot mix $(1+1)$ followed by pot mix alone.

\section{Hardening / acclimatization}

The experiment was taken up to study the effect of hardening media for acclimatization of the in vitro regenerated plantlets of Momordica cymbalaria. In the present study, pot mixture alone or in combination with cocopeat was found to be the most ideal hardening medium. The results of the present study are in line with those of Thiripurasundhari and Rao (2011) in Coccinia indica observed that in pot mixture alone or in combination with cocopeat gave the highest plant survival rates.

In conclusion the present study revealed that micropropagation through direct organogenesis using axillary bud could serve as a viable explant when MS medium is augmented with $2.0 \mathrm{mgl}^{-1}$ BAP for shoot regeneration. These shoots multiplied to 7.48 numbers in MS medium supplemented with $3.0 \mathrm{mgl}^{-1} \mathrm{BAP}$, followed by maximum rooting percentage in half MS medium fortified with $1.0 \mathrm{mgl}^{-1} \mathrm{IBA}+0.1 \mathrm{mgl}^{-1}$ of NAA and finally acclimatized in pot mixture, coco peat afford mass multiplication of Momordica cymbalaria

\section{References}

Debnath, B., Sinha,S. and Sinha, R.K. 2013. Rapid in vitro differentiation and regeneration of Momordica dioica Roxb. Indian J. Plant Sci., 2(3): 43-47.

Gomez, K.A and Gomez, A.A. 1984.Statistically procedure for agricultural research. An IRRI book, Wyley inter science application pulication, John Wyley and Sons, Newyork. USA. pp.680

Jeyadevi, R., Sivasudha, T., Rameshkumar, A., Sangeetha, B., Ananth, D.A. and Aseervatham, G.S.B. 2012. Nutritional constituents and medicinal values of Momordica cymbalaria. Asian Pacific J. Tropical Biomedicine.S456-S461.

Jeyakumar, J.J.,Kamaraj, M. and Thiruvengadam. 2013. Biochemical analysis of in vitro induced callus of Cucumis anguria L. Int. J. Pharm Bio 
Sci., 4(3): $484-489$.

Karim, M.A and Ahmed, S.U. 2010. Somatic embryogenesis and micro propagation in teasle Gourd. Int. J. Environ. Sci. Dev., 1(1): 10-14.

Mahzabin, F., Parvez, S. and Alam, M.F. 2008. Micropropagation of Cucurbita maxima Duch. through shoot tip culture. J. Bio-Sci.,16: 59-65.

Munsur, M. A. Z., Haque, M.S., Nasiruddin, K.M. and Hasan, M.J. 2007. Regeneration of bitter gourd (Momordica charantia L.) fromleaf segments and root tips. Prog. Agric., 18(2): 1-9.

Paravathi, S. and Kumar. V.J.F. 2002. Studies on chemical composition and utilization of wild edible vegetable athalakkai (Momordica tuberosa). Pl. Foods Hum Nutr., 57: 215-222.

Rathod, V. 2015. Plant regeneration in Momordica Dioica (Roxb) by root explant.J. Pharmacy and Bio Sci., 10(2): 80-83.
Sridhar, T.M. and Naidu, C.V. 2011. In Vitro Direct Shoot Organogenesis from Leaf Explants of Solanum nigrum (L.) An Important Antiulcer Medicinal Plant. J. Phytol., 3(5): 29-35.

Ugandhar, T., Venkateshwarrlu, M., Begum, G., Srilatha, T. and Jaganmohanreddy, K. 2011. In Vitro plant regeneration of Cucumber (Cucumis sativum (L.) from cotyledon and hypocotyl explants. Sci. Res. Rep., 1(3): 164-169.

Venkateshwarlu, M. 2009. Direct multiple shoots proliferation of muskmelon (Cucumis melo L.) from shoot tip explants. Int. J. Pharma and Biosci., 2(3): 645-652.

Verma, A.K., Kumar, M., Tarafdar, S., Singh, R. and Thakur, S. 2014. Development of protocol for micro propagation of gynoecious bitter gourd (Momordica charantia L). Int. J. Plant, Animal and Environ. Science.

\section{How to cite this article:}

Devi, T., V. Rajasree, V. Premalakshmi, K. Hemaprabha and Praneetha, S. 2017. In vitro Protocol for Direct Organogenesis in Momordica cymbalaria. Fenzl. Int.J.Curr.Microbiol.App.Sci. 6(4): 2392-2402. doi: https://doi.org/10.20546/ijcmas.2017.604.279 